\title{
Immunopathology
}

\section{SERUM FREE LIGHT CHAINS}

Karl W. Baumgart

Immunology, Douglass Hanly Moir Pathology, Sonic

Healthcare, NSW, Australia

Serum free light chain estimations entered most clinical laboratory test menus from September 2008. Their main clinical utility is in the identification of persons who might have a light chain only plasma cell dyscrasia. Other reasons clinicians request these assays are for the monitoring of persons with myeloma and monitoring progression of renal impairment. Increased sensitivity for detection of excessive free light chain production over urine protein electrophoresis and immunofixation may earlier identify persons with AL amyloid or myeloma. Non-compliance by patients with requests for urine EPG-IFE can be offset by serum free light chain estimations. Serum free light chain assays are unable to define clonality in a person with abnormal free light chain levels unlike serum or urine protein electrophoresis. Measurements can be insensitive to prozone effects, potentially underestimating light chain levels despite careful testing strategies. Some monoclonal free light chain bands may have idiosyncratic charge and other physical characteristics that can frustrate good assay systems. Physician responses to abnormal results may vary according to clinical context in terms of known monoclonal bands, renal impairment, suspicion of AL amyloid, immunosuppressive treatments. Robust and reliable serum free light chains have earned a mature role in serum protein studies with strong support by clinician users.

\section{LABORATORY DETECTION OF BIOLOGIC THERAPY}

Daman Langguth

SNP, Brisbane, Qld, Australia

Biologic drugs have revolutionised many medical conditions. Like all medicines there is a role for therapeutic drug monitoring (TDM) with some agents in some conditions. The utility of TDM with biologic agents will be explored, covering methods of detection as well as the evidence that TDM is effective or otherwise.

\section{BIOLOGICS IN IBD}

$\underline{\text { Susan Connor }}$

Liverpool Hospital, Liverpool, NSW, Australia

This talk will explain the current biologics and small molecules being used for inflammatory bowel disease, an increasingly prevalent disease in Australia, and the key role pathologists play in optimising biologic use for this patient population. The talk will also cover how we use biomarkers, particularly calprotectin, to facilitate IBD diagnosis and monitoring. The talk will be practical and simple.

\section{AUTOIMMUNE TOXICITIES OF T CELL CHECKPOINT INHIBITORS}

Sarah C. Sasson $^{1,2}$

${ }^{1}$ NSW Health Pathology, ICPMR Westmead Hospital, Sydney, NSW, Australia; and ${ }^{2}$ The Kirby Institute, University of New South Wales, Sydney, NSW, Australia

$\mathrm{T}$ cell checkpoint inhibitors $(\mathrm{CPI})$ are revolutionising the treatment of melanoma and other malignancies but come at the cost of immune related adverse events (irAE). Up to $55 \%$ of patients receiving combination anti-CTLA-4/PD-1 therapy develop Grade 3-4 irAE. These iatrogenic autoimmune toxicities can affect nearly every organ system, but most commonly present with disorders of the skin, endocrine organs and gastrointestinal tract. Clinical management of irAE commonly involves discontinuation of CPI and corticosteroids as first line immunosuppression. Second line agents include infliximab and mycophenolate, however there is a paucity of RCT data in this area. Our understanding of the pathogenesis of irAE remains rudimentary. These is no clear association with sex, age or genotype and the role of autoantibodies and the microbiome remains unresolved. CPI can be associated with exacerbations of previously diagnosed autoimmune disease, or de novo autoimmunity can develop. This presentation will take a particular focus on CPI-associated colitis, the irAE accounting for the greatest mortality. Recent studies into the cellular and molecular characteristics of CPI-associated colitis have identified novel strategies for more targeted therapeutics. The ongoing and successful use of CPI will need to be paired with improved detection, understanding and management of the significant off-target effects. 